\title{
Channel Estimation Enhancement in OFDM System based on Time Domain Interpolation
}

\author{
Mahdi Abdul Hadi \\ Asst. Professor \\ University of Baghdad \\ Electronic and Communication Engineering \\ Department
}

\author{
Omar Almukhtar Tawfeeq \\ MSc. Student \\ University of Baghdad \\ Electronic and Communication Engineering \\ Department
}

\begin{abstract}
In practical OFDM systems the transmitter send the data out through a multipath fading channel. At the receiver, the received data distorted by the channel characteristic. So, in order to recover the transmitted data, the channel effect must be estimated. In this paper channel estimation based on comb type pilot arrangement has been investigated by implementing least Square (LS) and Minimum Mean Error Square (MMSE) channel estimator to estimate the channel at the pilot locations. While the channel interpolation was done using linear interpolation, and time domain interpolation techniques. Also DFT-based estimation technique proposed to improve the performance of LS and MMSE estimator even more. Both LS and MMSE estimator have been implemented with and without DFT-based estimation technique and compare their performance by measuring the bit error rate BER with BPSK as modulation scheme and the channel is assumed to be multipath Rayleigh slow fading channel based on Clark's model.
\end{abstract}

\section{General Terms}

Performance enhancement, communication, DFT algorithms

\section{Keywords}

OFDM, MMSE, Inter-Carrier-Interference (ICI), Channel estimation, Rayleigh slow fading channel

\section{INTRODUCTION}

The growing demand of multimedia services, Internet contents and mobile communication lead to increase the interest to high speed communications. This requires a reliable communication system, wide bandwidth and efficient transmission techniques especially in wireless environment where the channel is very challenging. In Single-Carrier (SC) Transmission over multipath environment (fading channel), when increasing the data rate this leads to increase the intersymbol-interference (ISI). As a result this increase the error rate and low quality of service. To overcome this problem, Multi-Carrier (MC) transmission can be used instead. Orthogonal Frequency Division Multiplexing (OFDM) is Multiple-Carrier (MC) transmission method known for its capability to mitigate multipath effect. By transforming the frequency selective fading channel into many parallel flat fading channel [1]. Also channel estimation based on pilot insertion is used to estimate and equalize the effect of the fading channel to make the system more reliable [2] [3]. However, pilot insertion can be performed in two approach, either by inserting pilot tones into all subcarriers of the OFDM symbols with a specific period or inserting pilot tones into each OFDM symbol at periodically-located subcarriers.
The first one, the block type pilot channel estimation, has been developed under the assumption of fast varying channel within one OFDM symbol. The second one, the comb-type pilot channel estimation, has been introduced to satisfy the need for equalizing when the channel response changes slowly within the OFDM symbol and it is required less pilot tones than the block type, so we used the comp type pilot arrangement. On the other hand, one must take into account the ratio of pilot data to actual (real) data, so a trade-off should be made between OFDM signal performance and data transmission efficiency, therefore, its important to know how to and where to insert the pilot tones [4].

The reminder of this paper is organized as follows. After a brief description of the system model in Section 2, the conventional channel estimator using comb-type pilot is described in Section 3 and the DFT-based channel estimator presented in Section 4 respectively. The simulation results are given in Section 5.

\section{SYSTEM MODEL}

The OFDM system based on pilot channel estimation is given in Figure 1. First the input binary data mapped according to the type (M-PSK/M-QAM) of the signal mapper. And then, the mapped data converted from serial to parallel form, after that pilot tones inserted in appropriate locations to be used later for estimating the channel at those locations. After inserting pilot tones, N-point IFFT block used to transform the $\mathrm{S}(\mathrm{k})$ into time domain signal $\mathrm{s}(\mathrm{n})$ as follows.

$$
\begin{aligned}
\boldsymbol{s}(\boldsymbol{n}) & =\boldsymbol{I D F T}\{\boldsymbol{S}(\boldsymbol{k})\} \quad \boldsymbol{n}=\mathbf{1}, \mathbf{2}, \ldots, \boldsymbol{N}-\mathbf{1} \\
& =\sum_{k=0}^{N-1} S(k) e^{j(2 \pi k n / N)}
\end{aligned}
$$

Then guard interval can be inserted in two different ways. The first one is zero padding (ZP) that pads the guard interval with zeroes. The other way is cyclic extension of the OFDM symbol (for some continuity) to extend the OFDM symbol by copying the last samples of the OFDM symbol into its front. Guard interval length is chosen to be equal or longer than the maximum delay spread of the fading channel to reduce the effect of inter symbol interference ISI and inter carrier interference ICI. Let $N_{g i}$ is the length of the guard interval, and $s_{f}(n)$ is the resultant OFDM symbol after cyclic extension.

$s_{f}(n)= \begin{cases}s(N+n), & n=-N_{g i},-N_{g i}+1, \ldots,-1 \\ s(n), & n=0,1,2, \ldots, N-1\end{cases}$

The transmitted signal will pass through the frequency selective time varying fading channel with additive noise. The received signal is given by [5]. 


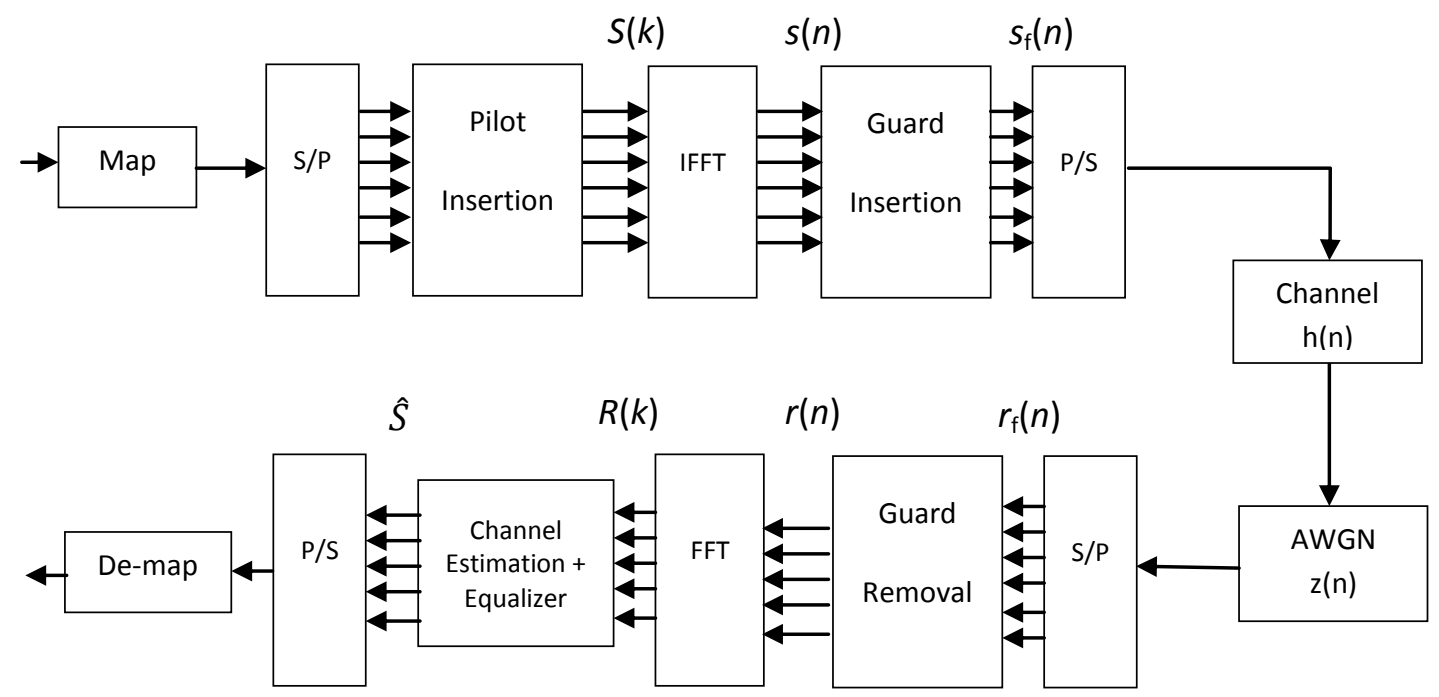

Fig 1: Basic OFDM system

$$
y_{f}(n)=s_{f}(n) * h(n)+z(n)
$$

Where $z(n)$ is Additive White Gaussian Noise (AWGN) and $h(n)$ is the channel impulse response. The channel response can be represented by.

$$
\begin{gathered}
h(t)=h_{r e}(t)+j h_{i m}(t) \\
h_{r e}(t)=\frac{1}{\sqrt{M}} \sum_{n=1}^{N} \cos \left(2 \pi f_{d} t \cos \left(\alpha_{n}\right)+\emptyset_{n}\right) \\
h_{i m}(t)=\frac{1}{\sqrt{M}} \sum_{n=1}^{N} \sin \left(2 \pi f_{d} t \cos \left(\alpha_{n}\right)+\emptyset_{n}\right)
\end{gathered}
$$

Where $h_{r e}(t)$ is the real part of $h(t), h_{i m}(t)$ is the imaginary part of $h(t), M$ is the number of propagation paths, $f_{d}$ is the maximum Doppler shift, $\alpha_{n}$ and $\varphi_{n}$ are respectively, the angle of arrival and initial phase of the $m$ th propagation path .Both $\alpha_{n}$ and $\varphi_{n}$ are uniformly distribute over $[-\pi, \pi]$ for all $m$ and they are mutually identically independent distribution (i.i.d) [6].

Now at the receiver side the same process is applied to the received signal but in reversed order and also the channel estimation and equalization block is used to estimate and equalize the channel effect.

\section{CONVENTIONAL CHANNEL ESTIMATION AT PILOT FREQUENCIES IN COMP TYPE PILOT ARRANGEMENT}

In comb-type pilot arrangement as shown in figure 2, the pilot signals are uniformly inserted into $X(k)$ according to the following equation:

$$
\begin{gathered}
S(k)=S(m L+l) \\
= \begin{cases}s_{p}(m), & l=0 \\
\text { info, } & l=1, \ldots, L-1\end{cases}
\end{gathered}
$$

Where $L=$ number of carriers $/ N_{p}$ and $s_{p}(m)$ is the $m$ th pilot carrier value, then $\left\{H_{p}(k) \quad k=0,1, \ldots, N_{p}\right\}$ defined as the channel frequency response at pilot sub-carriers. Then channel estimation at pilot sub-carriers based on Least Square (LS) estimation is given by:

$$
H_{e}(k)=\frac{R_{p}(k)}{S_{p}(k)} \quad, k=0,1, \ldots, N_{p}-1
$$

Where $R_{p}(k)$ and $S_{p}(k)$ are output and input data at the $k$ th pilot sub-carrier respectively [5].

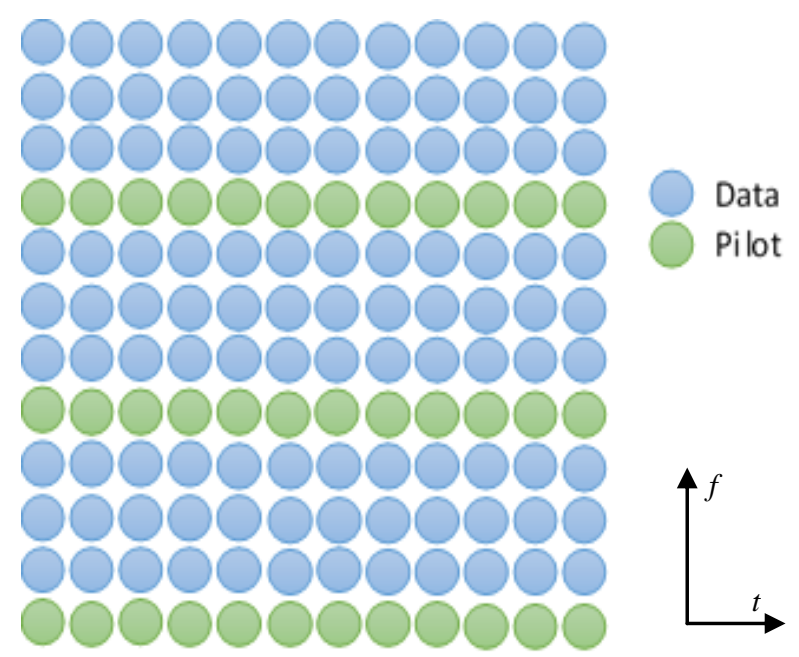

Fig 2: comp type pilot arrangement

Then the transfer function for non-pilot subcarriers can be obtained by adjacent pilot subcarriers channel estimation result via interpolation. There are various interpolation algorithms performed base on different pilot patterns. The most simple and most extensively adopted interpolation algorithm is linear interpolation, which just take the two neighboring pilots at the same time slot into, performing linear interpolation to get non-pilot subcarrier channel estimation as follows.

Suppose the frequency interval of the neighboring pilot subcarrier is $L, l(l=1,2, \ldots, L-1)$ is the index of the non-pilot subcarrier between two adjacent pilots, the $m$ th and $(m+l) t$ h pilots. Thus the transfer function for non-pilot subcarriers can be linearly expressed in terms of the subcarrier index as 


$$
\widehat{H}=\widehat{H}_{m L+l}=\left(1-\frac{l}{L}\right) \widehat{H}_{m}+\left(\frac{l}{L}\right) \widehat{H}_{m+1}
$$

Then, after the equalizer in figure 1 :

$$
\hat{S}(k)=\frac{R(k)}{\widehat{H}(k)}
$$

Where,

$$
R(k)=S(k) H(k)+Z(K)
$$

Sub equation (8) in (9) then,

$$
\begin{aligned}
& \hat{S}(k)=\frac{S(k) H(k)+Z(K)}{\widehat{H}(k)} \\
& \hat{S}(k)=\frac{S(k) H(k)}{\widehat{H}(k)}+\frac{Z(K)}{\widehat{H}(k)}
\end{aligned}
$$

Due to the second part of equation (10), the LS estimator is subject to noise enhancement specially when the channel in deep fading.

Now, consider the channel in equation (7) after LS estimation and linear interpolation. Using the weight matrix $W$, define $\widetilde{H}=W \widehat{H}$, which corresponds to the MMSE estimation. Referring to figure 3, the Mean Square Error (MSE) of the estimated channel $\widetilde{H}$ is given as

$$
\begin{aligned}
M S E= & E\left\{|e|^{2}\right\}=E\left\{|H-W \widehat{H}|^{2}\right\} \\
& =E\left\{|H-\widetilde{H}|^{2}\right\}
\end{aligned}
$$

Where,

$$
W=R_{H \widehat{H}} R_{H \widehat{H}}^{-1}
$$

Where $R_{H \widehat{H}}$ is the cross-correlation between the true channel vector and LS estimated channel vector, $R_{\hat{H} \widehat{H}}$ is the autocorrelation matrix of $\widehat{H}$. Then MMSE channel estimation defined as

$$
\widetilde{H}=\left(R_{H \widehat{H}} R_{H \widehat{H}}^{-1}\right) \widehat{H}
$$

\section{Fig 3: MMSE channel estimation}

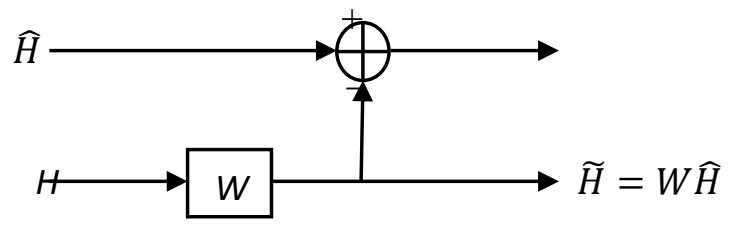

Then, the MMSE channel estimation method shows better estimate in terms of $W$ in such a way that the MSE in equation (11) is minimized [7]

\section{IMPROVED LS AND MMSE ESTIMATORS USING DFT-BASED ESTIMATION TECHNIQUE}

The DFT-based channel estimation technique has been introduced to improve the performance of LS or MMSE channel estimation by eliminating the effect of noise outside the maximum channel delay. The noisy channel estimation (in frequency domain) transformed to the time domain by an IDFT, and then transformed back to the frequency domain by a DFT after appropriately being processed in order to reduce noise effects [8]. As shown in figure 4, DFT-based channel estimation with comb-type pilot proceeds as following:

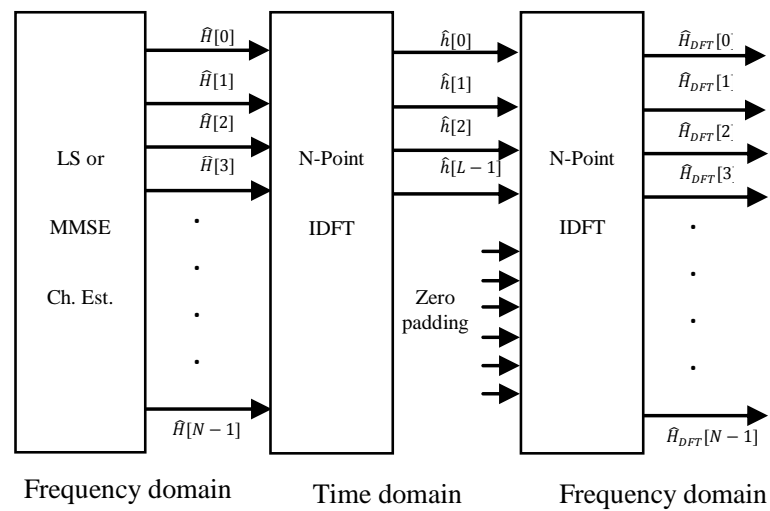

Fig 4: DFT-based channel estimation

Step1: Let $\widetilde{H}(k)$ denote the estimated channel gain at the $k t h$ subcarrier, obtained by either $L S$ or $M M S E$ channel estimator. Taking the IDFT to the estimated channel.

$$
\operatorname{IDFT}\{\widetilde{H}(k)\}=\sum_{k=0}^{N-1} \widetilde{H}(k) e^{j\left(\frac{2 \pi k n}{N}\right)}
$$

Where $z[n]$ denotes the noise component in the time domain. Step2: the processing in the time domain is applied to reduce the noise effect. The most channel power concentrates on only small parts of the estimated time-domain samples that are transformed by IDFT .A straight forward way is to ignore (zero padding) the coefficients ,called as the non-significant taps, which contain more noise than channel power and only transform the remaining coefficients, called as the significant taps,

$$
h(n)= \begin{cases}h(n) \quad, n=0,1, \ldots, \beta \\ 0 \quad, n=\beta+1 \ldots, N-1\end{cases}
$$

Usually the window length $\beta \leq L$ can be set as the sampled maximum excess delay of the channel.

Step3: take N-point DFT of the estimated channel impulse response after ignoring the non-significant taps to obtain the estimated channel frequency response of length- $\mathrm{N}$

$$
\widetilde{H}_{D F T}(\mathrm{k})=\frac{1}{N} \sum_{k=0}^{N-1} h(n) e^{-j\left(\frac{2 \pi k n}{N}\right)}
$$

\section{SIMULATION AND RESULTS}

The setting parameter for OFDM system used in the simulation are indicated in table 1 .

Table 1. Simulation parameter

\begin{tabular}{|c|c|}
\hline Parameter & Value \\
\hline FFT size (total carriers) & 64 \\
\hline Guard interval & $16(25 \%$ FFT size $)$ \\
\hline Guard type & Cyclic extension \\
\hline Pilot carriers & $4,8,16$ \\
\hline Signal constellation & BPSK \\
\hline Channel moddel & $\begin{array}{c}\text { Rayleigh fading (ITU-R } \\
\text { Pedestrian A Model) }\end{array}$ \\
\hline
\end{tabular}

Under the assumption of having perfect synchronization and since the aim is to observe channel estimation performance. Moreover, guard interval was chosen to be greater than the maximum delay spread in order to avoid inter-symbol interference. Simulations are carried out for different signalto-noise (SNR) ratios and for different number of pilots. The multi-path fading channel model used in the simulation is 
Rayleigh fading (ITU-R Pedestrian A Model) [9] described in the Power Delay Profile (PDP) in table 2.

Table 2, Power delay profile for ITU-R Pedestrian A Model

\begin{tabular}{|c|c|c|}
\hline Tap & Relative delay (ns) & Relative power $(\mathrm{dB})$ \\
\hline 1 & 0 & 0 \\
\hline 2 & 110 & -9.7 \\
\hline 3 & 190 & -19.2 \\
\hline 4 & 410 & -22.8 \\
\hline
\end{tabular}

Also LS method was used to estimate the channel at the pilot frequencies. And then to estimate the channel between the pilot frequencies by linear interpolation. LS estimator description is given in section 3. To minimize the MSE of this estimator, MMSE channel estimation was used as shown in figure 3. From BER performance of LS and MMSE channel estimation for 8 and 16 pilots shown in figure 5(a-b) respectively, it is obvious that the LS estimator is higher than MMSE estimator by $10-20 \mathrm{~dB}$ in figure $5(\mathrm{a})$, and by $7-10 \mathrm{~dB}$ in figure 5(b), This is because the LS estimator is subject to noise enhancement especially when the channel in deep fading. The performance of estimation can be improved by increasing the number of pilots. But on the other hand, increasing pilot tone reduces data transmission efficiency of the OFDM systems.

To improve the performance of the LS and MMSE even more without increasing the pilots, DFT-based channel estimation technique has been used, and it shows a noticeable improvement for both LS and MMSE channel estimators, as shown in figure 6 .

Finally figure $7(\mathrm{a}, \mathrm{b}, \mathrm{c}, \mathrm{d})$, shows the estimated channel frequency response for LS and MMSE both with and without DFT estimation technique verses the true channel. It is obvious from figure 7(a) that LS estimator fails to track the channel in deep fading, farther improvement using MMSE estimator in figure 7(c). but there is a noticeable improvement on both LS and MMSE when using DFT technique as shown in figure 7(b),7(d) respectively.

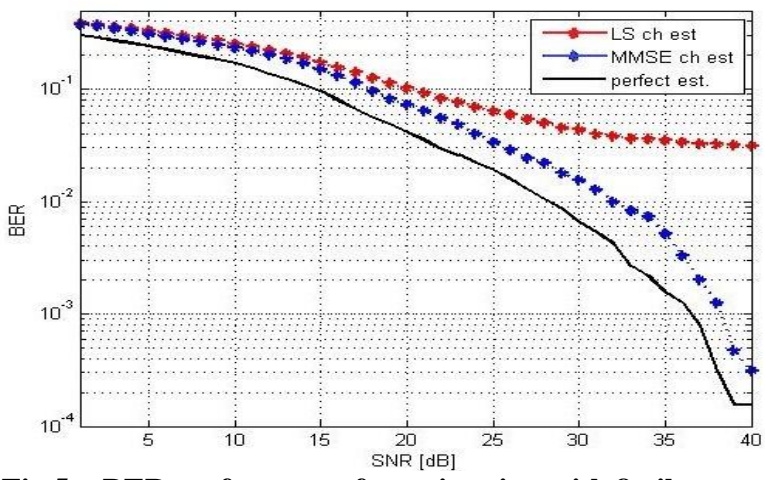

Fig 5a: BER performance for estimation with 8 pilot tons and $30 \mathrm{~km} / \mathrm{h}$ speed

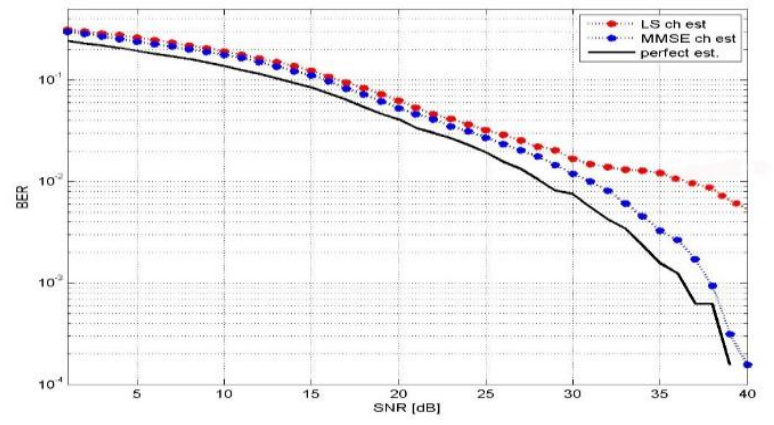

Fig 5b: BER performance for estimation with 16 pilot tons and $30 \mathrm{~km} / \mathrm{h}$ speed

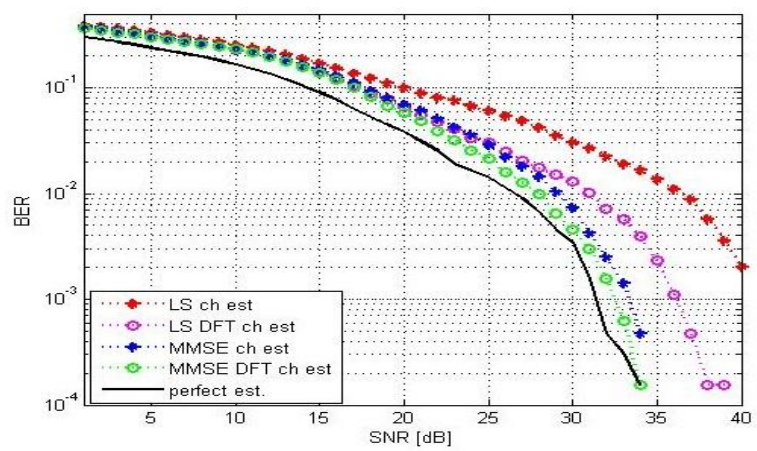

Fig 6: BER performance of LS and MMSE both with and without DFT estimation technique
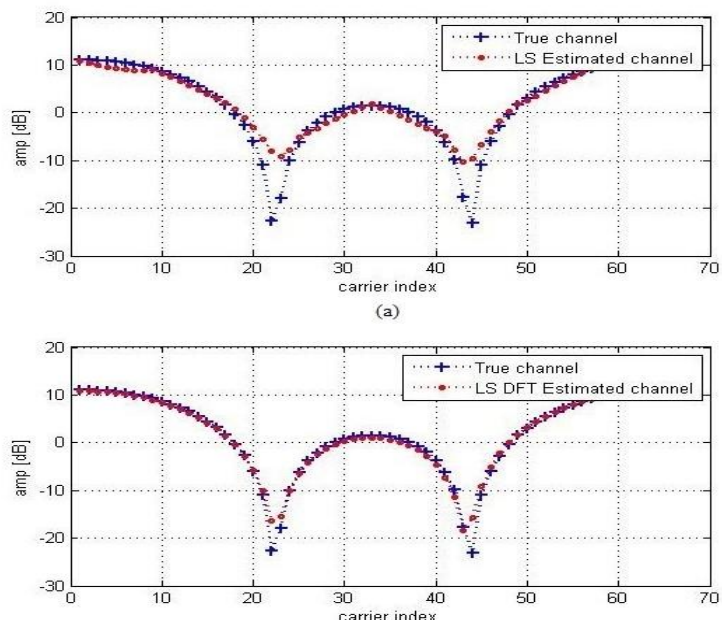

(b)

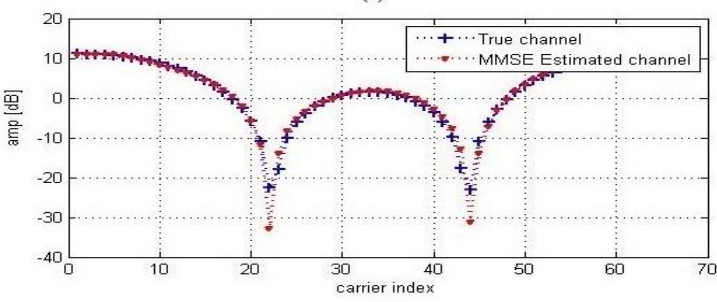

(c)

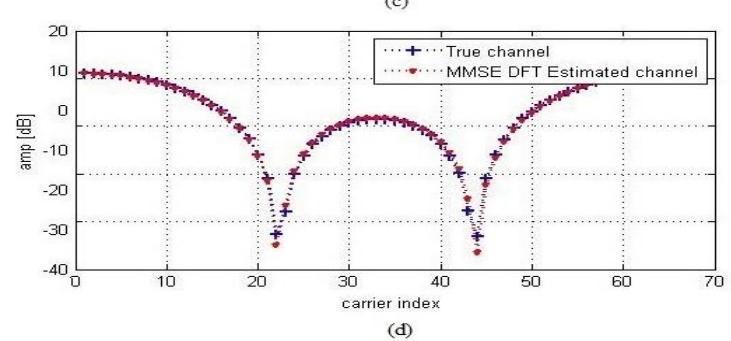

(d) 


\section{CONCLUSION}

The performance of the most popular channel estimators based on comp type pilot arrangement in OFDM system (the LS and MMSE) has been investigated. MMSE estimator shows butter performance than LS estimator due to its ability to minimize the MSE using the weight matrix $\mathrm{W}$, farther improvement on LS and MMSE estimators using DFT-based estimation technique due to the ability of the method to eliminate the noise outside the maximum channel delay. Fore future research, multi iterative procedure can be applied to enhance the performance of DFT-based estimation technique by repeating the three steps in section 4 more the once and compare the mean square error MSE to a pre-assigned threshold value

\section{ACKNOWLEDGMENTS}

Our thanks to the experts who have contributed towards development of this paper.

\section{REFERENCES}

[1] Abdelhakim Khlifi, Ridha Bouallegue, "Performance Analysis of LS and LMMSE Channel Estimation Techniques for LTE Downlink Systems," International Journal of Wireless \& Mobile Networks (IJWMN) Vol. 3, No. 5, October 2011

[2] Jung-Chieh Chen, Chao-Kai Wen, Pangan Ting, "An Efficient Pilot Design Scheme for Sparse Channel Estimation in OFDM Systems," IEEE Communication Letters, VOL. 17, NO. 7, JULY 2013

[3] R. Negi and J. Cioffi, "Pilot tone selection for channel estimation in a mobile OFDM system," IEEE
Transactions on Consumer Electronics, Vol. 44, No. 3, AUGUST 1998

[4] Lilong Liu, Xuelin Yang, Jun Li ; Meihua Bi, Hao He, Weisheng $\mathrm{Hu}$, "Experimental evaluation of pilot arrangement for channel estimation in OFDM systems," IEEE Communications and Photonics Conference and Exhibition, 2011. ACP. Asia, Page(s): $1-6$

[5] Coleri, S., Ergen, M., Puri, A., and Bahai, A., "Channel estimation techniques based on pilot arrangement in OFDM systems," IEEE Transactions on Broadcasting, Vol. 48, No. 3, SEPTEMBER 2002, 223-229.

[6] Chengshan Xiao, Zheng, Y.R., Beaulieu, N.C. "Novel Sum-of-Sinusoids Simulation Models for Rayleigh and Rician Fading Channels," IEEE Transactions on Wireless Communications, VOL. 5, NO. 12, DECEMBER 2006.

[7] Yong Soo Cho, Jaekwon Kim, Won Young Yang, Chung G. Kang (2010). MIMO-OFDM Wireless Communications with MATLAB. Asia: John Wiley \& Sons Pte Ltd.

[8] Xu Yan , Lin Ying, "Research of OFDM systems channel estimation based on DFT improved algorithm," IEEE 4th International Conference on Computer Science \& Education, 2009. ICCSE '09. Page(s) 250-253.

[9] Recommendation (1997) ITU-R M.1225. Guidelines for Evaluation of Radio Transmission Technologies for IMT-2000. 